\title{
The Minimum Wind Speed for Sustainable Turbulence in the Nocturnal Boundary Layer
}

\author{
B. J. H. VAn DE Wiel, $*$ A. F. Moene, ${ }^{+}$H. J. J. Jonker, ${ }^{\#}$ P. BAAs, ${ }^{@}$ S. BAsU, ${ }^{\&}$ J. M. M. DondA, ${ }^{*}$ \\ J. Sun, ${ }^{* *}$ AND A. A. M. HOLTSLAG ${ }^{+}$ \\ * Fluid Dynamics Laboratory, Eindhoven University of Technology, Eindhoven, Netherlands \\ ${ }^{+}$Department of Meteorology and Air Quality, Wageningen University, Wageningen, Netherlands \\ \# Applied Physics, Delft University of Technology, Delft, Netherlands \\ @ Atmospheric Research Division, Department of Climate and Seismology, Royal Netherlands Meteorological \\ Institute (KNMI), De Bilt, Netherlands \\ \& Department of Marine, Earth and Atmospheric Sciences, North Carolina State University, Raleigh, North Carolina \\ ** National Center for Atmospheric Research, Boulder, Colorado
}

(Manuscript received 30 March 2012, in final form 2 July 2012)

\begin{abstract}
The collapse of turbulence in the nocturnal boundary layer is studied by means of a simple bulk model that describes the basic physical interactions in the surface energy balance. It is shown that for a given mechanical forcing, the amount of turbulent heat that can be transported downward is limited to a certain maximum. In the case of weak winds and clear skies, this maximum can be significantly smaller than the net radiative loss minus soil heat transport. In the case when the surface has low heat capacity, this imbalance generates rapid surface cooling that further suppresses the turbulent heat transport, so that eventually turbulence largely ceases (positive feedback mechanism). The model predicts the minimum wind speed for sustainable turbulence for the so-called crossing level. At this level, some decameters above the surface, the wind is relatively stationary compared to lower and higher levels. The critical speed is predicted in the range of about 5$7 \mathrm{~m} \mathrm{~s}^{-1}$, depending on radiative forcing and surface properties, and is in agreement with observations at Cabauw. The critical value appears not very sensitive to model details or to the exact values of the input parameters. Finally, results are interpreted in terms of external forcings, such as geostrophic wind. As it is generally larger than the speed at crossing height, a $5 \mathrm{~m} \mathrm{~s}^{-1}$ geostrophic wind may be considered as the typical limit below which sustainable, continuous turbulence under clear-sky conditions is unlikely to exist. Below this threshold emergence of the very stable nocturnal boundary layer is anticipated.
\end{abstract}

\section{Introduction}

A good understanding of nocturnal boundary physics is relevant for various applications related to weather and climate. Although considerable progress has been reported in this field of boundary layer (BL) research, a fully comprehensive framework valid for all types of nocturnal boundary layers (NBLs) appears to be lacking (Derbyshire 1999b; Fernando and Weil 2010; Baklanov et al. 2011). Nevertheless, it seems to be useful to classify stable boundary layers into two major prototypes (Mahrt et al. 1998) — the weakly stable boundary layer (WSBL)

Corresponding author address: B. J. H. Van de Wiel, Fluid Dynamics Laboratory, Eindhoven University of Technology, P.O. Box 513, 5600 MB Eindhoven, Netherlands.

E-mail: b.j.h.v.d.wiel@tue.nl and the very stable boundary layer (VSBL) — based on their generally observed characteristics.

The WSBL is generally characterized by the presence of continuous turbulence, and it tends to occur in windy and/or cloudy conditions. It is relatively well understood and turbulent transport can be described satisfactorily using local similarity scaling (Nieuwstadt 1984; Derbyshire 1990; Sorbjan 2006; Basu et al. 2006; Beare et al. 2006; Cuxart et al. 2006; Svensson et al. 2011). The VSBL, in contrast, is less well understood (Poulos et al. 2002; Fernando and Weil 2010). It is characterized by strong surface inversions, as it prevails in situations with weak winds and clear skies (Edwards 2009). The strong stratification causes turbulence to be either very weak or even virtually absent (Mahrt 2011). At the same time, the relatively calm background state may be suddenly interrupted by sudden events of turbulent mixing, which 
may lead to so-called intermittent bursts (Sun et al. 2003).

From a practical viewpoint, the aforementioned diagnostic seems both attractive and useful in order to organize observational data. From a theoretical perspective, however, one would like to predict whether a particular night will manifest itself as a VSBL or as a WSBL under given external, synoptic conditions. According to the interpretation above, it is the presence or absence of sustained turbulence that finally separates the two prototype regimes. The goal of the present study is therefore to predict the critical synoptic conditions for sustained turbulence as a proxy to predict the WSBLVSBL transition.

At night radiative surface cooling is competing with the ambient wind shear, which tries to maintain turbulence mixing. The relative magnitude of those processes will ultimately decide on the presence or absence of turbulence. In turn, those processes are externally driven by the incoming longwave radiation at the top of the boundary layer, which is mainly dictated by the presence or absence of clouds, and by the synoptic pressure gradient (Van de Wiel et al. 2002a; Svensson et al. 2011). If we restrict ourselves to clear-sky cases, then the magnitude of the horizontal pressure gradient becomes decisive on the emergence of stable boundary layer regimes. Indeed, the appearance of various stable boundary layer (SBL) regimes in response to changing geostrophic wind magnitudes has been reported in climatological analysis on observations by, for example, Nieuwstadt (1984, section 1), Poulos et al. (2002), and Van de Wiel et al. (2012, hereafter VDW). Qualitatively similar behavior has been reported in stratified wind-tunnel experiments by Ohya et al. (1997), who found dramatic changes in boundary layer characteristics in response to decreasing mechanical forcing. Likewise, regime transitions have been modeled with simple 1D models (Estournel and Guedalia 1985; Revelle 1993; McNider et al. 1995; Derbyshire 1999a; Van de Wiel et al. 2002a; Walters et al. 2007; Acevedo et al. 2012) in large-eddy simulation (LES) studies (Jiménez and Cuxart 2005; Zhou and Chow 2011) and in studies using direct numerical simulations (Nieuwstadt 2005; Flores and Riley 2011).

In a companion paper (VDW), the mechanism behind the collapse of turbulence was investigated from theoretical analysis on a cooled channel flow. It was shown that collapse is essentially caused by the fact that the sustainable heat flux in stratified boundary layers is limited to a maximum under given mechanical forcing. The existence of such a maximum can be anticipated by the fact that turbulent heat transport in the nocturnal boundary layer will vanish for both very small (gradient

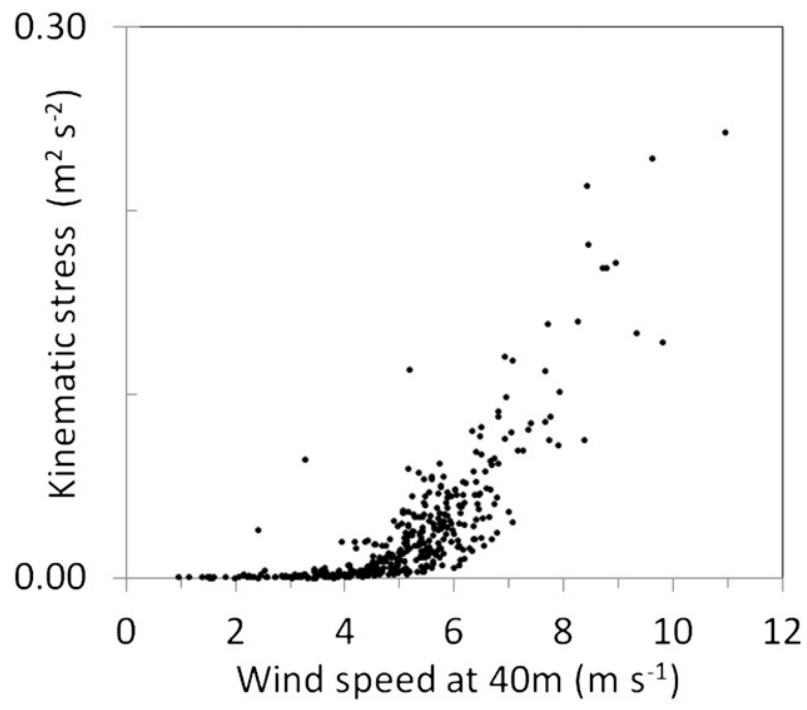

FIG. 1. Nighttime kinematic turbulent stress (at $5 \mathrm{~m}$ ) as a function of wind speed at $40 \mathrm{~m}$ for clear-sky situations observed at the KNMI Cabauw observatory. Each point represents a 4-h mean value. At nighttime a minimum wind speed is needed in order to generate significant stress values.

limited) and large temperature gradients (mixing limited; De Bruin 1994). For cases with strong geostrophic winds, turbulent heat fluxes are large enough to compensate the radiative cooling at the surface. However, in case of weak mechanical forcing, even the maximum heat flux may be too small compared to radiative cooling. In that case, temperature inversions may rapidly increase over surfaces with small heat capacity, so that turbulence becomes largely suppressed by the intense density stratification.

In the present study, the theoretical results of VDW are translated into a conceptual model. Emphasis lies on simplicity and practical applicability rather than on theoretical rigorousness as in VDW. Nevertheless, it can be shown that an estimate for the critical geostrophic wind speed for continuous turbulence can be given by considering first-order system feedbacks. As a first step, the framework will be formulated in terms of the wind speed at the so-called velocity crossing point. As this intermediate level, typically some decameters above the surface, the magnitude of the wind is usually more stationary than at lower or higher levels. It is, for example, known that near-surface winds have a strong tendency to weaken in the evening, whereas high-level winds tend to accelerate due to inertial effects (section 2). In Fig. 1 the sustainability of turbulence in response to mechanical forcing at crossing height is illustrated using observations from the Royal Netherlands Meteorological Institute (KNMI) Cabauw observatory (section 3). To this end the typical response of kinematic turbulent 
stress is given as a function of the magnitude of the wind at the crossing height-in this case, at about $40 \mathrm{~m}$ (VDW). From a 10-yr dataset, a subset of nights with clear skies and relatively stationary forcing conditions was selected. Each point represents an average of $4 \mathrm{~h}$ after sunset. It appears that a minimum wind speed is required in order to maintain a significant level of turbulence. The minimum wind speed at $40 \mathrm{~m}$ appears to be around $5 \mathrm{~m} \mathrm{~s}^{-1}$ for Cabauw. Interestingly, the existence of such wind speed threshold has also been reported for Cooperative Atmospheric-Surface Exchange Study 1999 (CASES99) data by Sun et al. (2012). Additionally, their analysis suggests a logarithmic relation between the threshold and the height of the wind observation (section 4).

The consequence of Fig. 1 in terms of geostrophic forcing can be anticipated: if $5 \mathrm{~m} \mathrm{~s}^{-1}$ at $40 \mathrm{~m}$ is insufficient to maintain nocturnal turbulence under clear skies at Cabauw, then this is certainly true for the geostrophic wind, which exceeds the magnitude of the wind at crossing level.

Following this rationale, first, a physical model for the relation depicted in Fig. 1 is formulated. Then, by assuming that the wind at the crossing point (CP) is generally lower than the geostrophic wind, one can derive a lower bound for the critical geostrophic wind. From this we classify nocturnal boundary layers in the external parameter and predict under which synoptic conditions the onset of the very stable boundary layer is foreseen at a certain location.

The paper is organized as follows. The model setup is given in section 2 . In section 3 predictions that use the concept of velocity crossing point are compared with Fig. 1. A physical interpretation of the results is given in section 4. Results are translated in terms of external forcing parameters in section 5 , and the classification of nocturnal boundary layers is compared to a few existing classifications from the literature. Finally, a discussion on the relevance of surface boundary conditions is given in section 6 , followed by concluding remarks.

\section{Model setup}

A conceptual bulk model is introduced as a simplification of complex reality. The purpose is to explain the mechanism for the collapse of turbulence in the nocturnal boundary from basic interactions. Comparison between such approach and formal theoretical analysis in VDW showed that the essence of the instability mechanism remains largely unchanged by such modifications.

First, the mechanical forcing is considered. It is well known that high-level winds (say, $>100 \mathrm{~m}$ ) tend to accelerate in the evening (Blackadar 1957; Banta 2008; Shapiro and Fedorovich 2010; Baas et al. 2012). Low-level

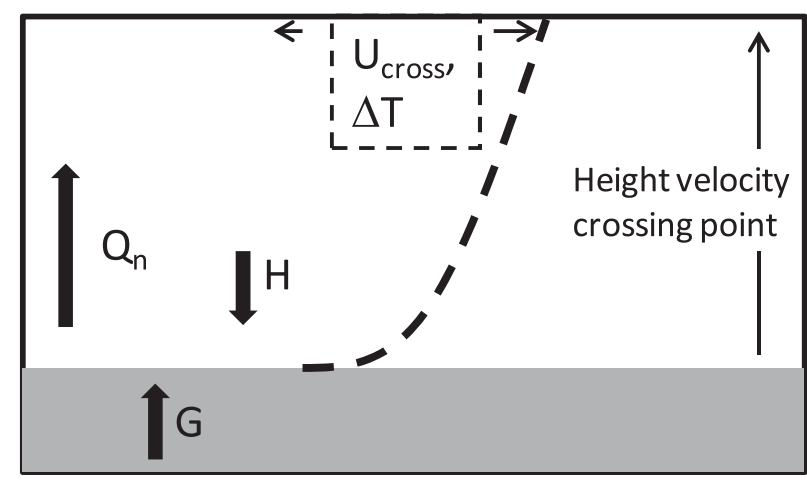

FIG. 2. Schematic picture of the bulk model. Wind speed at the velocity crossing point $U_{\text {cross }}$ is assumed to be constant. Net radiative cooling at the surface $Q_{n}$ is prescribed. The soil heat flux $G$ and the turbulent heat flux $H$ are modeled in terms of the bulk temperature inversion $\Delta T$.

winds (say, $<20 \mathrm{~m}$ ) usually weaken during this period (e.g., Lapworth 2008). Consequently, the magnitude of the wind has a tendency to remain constant at intermediate levels (say, between $\sim 30$ and $60 \mathrm{~m}$ ). As before, this level is referred to as the CP (VDW), or as the "reversal" height (Wieringa 1989). Theoretical analysis by VDW revealed that the existence of such a CP follows from momentum conservation principles in the case when diffusion time scales are smaller than acceleration time scales (as in the NBL). Mathematically, the $\mathrm{CP}$ can be seen as a velocity boundary condition for the lower SBL. Therefore, a mechanical forcing is imposed by prescribing a constant wind speed at the CP level (Fig. 2). From wind observations at the 200-m Cabauw tower, the CP appeared typically around $40 \mathrm{~m}$ (VDW). This will be used below.

The cooling of the system is driven by a constant, prescribed value of the net longwave radiation $Q_{n}$ at the surface. Formally, $Q_{n}$ cannot be truly constant, as it depends on the surface temperature itself. In contrast, observations show that upward longwave radiation indeed tends to decrease during the night due to surface cooling, but that this decrease is usually largely compensated by a similar decrease in incoming radiation (Derbyshire 1999a; Brunt 1941). The net radiation thus has a tendency to remain more or less constant in magnitude [e.g., Fig. 4 in Mahrt et al. (1998) and examples in Van de Wiel et al. (2003)]. The night average value is then mainly determined by the amount of low-level clouds present. We note that alternatively the so-called isothermal net radiation $Q_{i}$ (Monteith 1981; Holtslag and De Bruin 1988; Van de Wiel et al. 2002a) could be used as forcing, as it solely depends on external radiative parameters such as clear-air emissivity and cloud cover. However, additional model assumptions are needed to infer $Q_{i}$ from $Q_{n}$, whereas the latter is directly available 
from observations, so that this is preferred as the most practical alternative.

Here, surfaces with relatively low heat capacity are considered. Examples are snow-covered surfaces or surfaces with short grass. The storage term in the energy balance is then relatively small and will be discarded in order to simplify analysis. We also consider a dry atmosphere such that latent heat fluxes can be ignored. In equilibrium, the net radiation minus the soil heat flux $G$ must be balanced by the turbulent heat flux $H\left(\mathrm{~W} \mathrm{~m}^{-2}\right)$, defined as

$$
Q_{n}-G=H
$$

All fluxes are defined positive here. In VDW it was shown that the turbulent heat supply is bounded by a maximum value: both at weak (gradient limited) and strong inversions (mixing limited), the heat flux tends to zero. Under certain conditions even this maximum supply can be insufficient to meet the demand $Q_{n}-G$. This usually occurs with clear skies and weak winds. In the case when the surface has a low heat capacity, the imbalance will cause rapid surface cooling. This suppresses the turbulent heat transport even further, so that eventually turbulence will largely tend to cease (positive feedback mechanism). Note that in reality, some moderation of this feedback is expected from longwave radiative heat transfer processes (Edwards 2009; Derbyshire 1999a) that generally counteract the formation of extreme temperature inversions. However, the present authors argue that the basic qualitative mechanism for regime transition remains largely unchanged, as those effects become increasingly important after the transition to the VSBL has been established.

Historically, the above-mentioned line of reasoning based on the assumption of fixed shear has already been described by, for example, Derbyshire (1999a) and Delage et al. (2002). However, as correctly mentioned by Derbyshire, the validity of an ad hoc fixed bulk shear assumption is far from obvious for atmospheric flows: at first instance, the assumption seems to be invalid in pressure-driven flows, where the collapse of turbulence implies reduced boundary layer friction and hence invokes flow acceleration [good examples are given in Schubert (1977); see also Businger (1973)]. Additionally, the fixed-shear approach itself is highly sensitive to the magnitude of the wind speed (section 3; Delage et al. 2002), which would further disqualify the assumption. In this perspective, the recent notion by VDW that midlevel wind speeds have an initial tendency to remain constant is of crucial importance: they found that in the evening, boundary layer diffusion processes usually act much faster than the aforementioned pressure acceleration effects. With rapidly diffused momentum in the vertical, an initial acceleration due to reduced stress divergence at higher levels invokes a deceleration at lower levels (hence, some kind of crossing level in between). The bulk shear between the surface and the crossing point remains finite during this period, that is, at least below a bulk shear value that would be based on, say, a geostrophic speed at this level. Then, only later during the night, may the aforementioned flow accelerations due to pressure gradient effects become important. As such, the fixed bulk shear assumption seems to be plausible in the first hours after sunset.

Nevertheless, the above-mentioned mechanism remains a crude simplification. Besides, a total collapse of turbulence, in the sense that the flow ends up in a purely laminar state, is unlikely (Galperin et al. 2007). Rather, the aforementioned feedback will act as a precursor to a "new," very stable boundary layer regime with some weak residual turbulence (Mauritsen and Svensson 2007; Zilitinkevich et al. 2008).

To evaluate for which conditions a balance in Eq. (1) can or cannot be achieved, $G$ and $H$ need to be expressed in terms of the prefixed bulk wind shear $U_{\text {cross }}$ and the (yet unknown) temperature inversion $\Delta T$ over this layer.

Formally, the actual $G$ depends on the entire history of soil temperature (Heusinkveld et al. 2004; Edwards 2009). Its prediction would therefore involve a full description of subsurface temperature dynamics and its history, which is clearly beyond the scope of the present study. The simplest alternative would be to express $G$ as a fixed fraction of $Q_{n}$. The rationale behind such closure is the fact that nights with strong radiative heat loss tend to have cold surface temperatures that facilitate large fluxes from the underlying soil. This option is used in our interpretation (section 4). Here, we choose for intermediate complexity and parameterize the soil heat flux via

$$
G=\lambda \Delta T,
$$

with $\lambda$ as an empirical proportionality constant. According to Eq. (2), stronger temperature inversions tend to coincide with larger soil heat flux transport. In the literature, $G$ is often related directly to the temperature jump over the vegetation layer itself. In that case, $\lambda$ typically ranges between 3 and $8 \mathrm{~W} \mathrm{~m}^{-2} \mathrm{~K}^{-1}$ for short vegetated surfaces, such as grassland (Duynkerke 1999; Van de Wiel et al. 2003; Steeneveld et al. 2006). In many practical situations, however, direct detailed information on soil and vegetation temperatures are missing. Therefore, Eq. (2) expresses the soil heat flux in terms of a model parameter $\Delta T$. Hereby, we follow arguments by Van de Wiel et al. (2002b), who state that both the temperature jump over the vegetation layer and the jump over the bulk layer $\Delta T$ are mainly dominated by the amplitude of surface temperature dynamics [more details are found in Donda et al. (2012)]. 

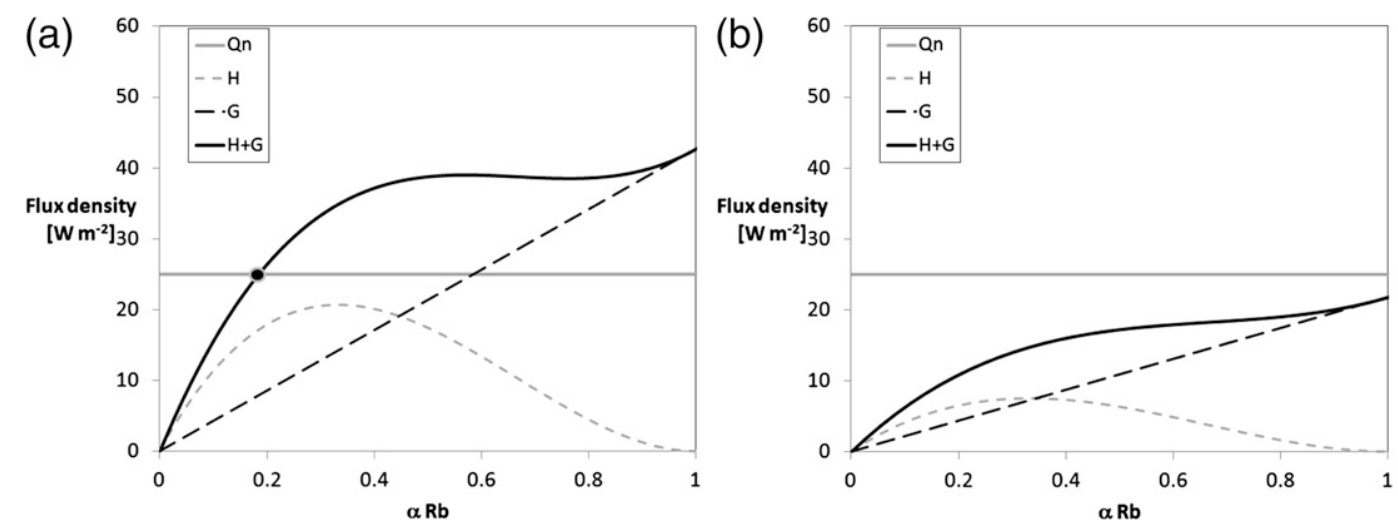

FIG. 3. Dependence of the surface energy balance terms on the bulk Richardson number showing (a) $U_{\text {cross }}=7$ and (b) $U_{\text {cross }}=5 \mathrm{~m} \mathrm{~s}^{-1}$. For the stronger wind case, a surface energy balance is reached at $\alpha R_{b} \approx 0.18$. For the weak wind case, the sum of turbulent and soil heat fluxes is too small to compensate for the radiate heat loss.

To relate turbulent fluxes to bulk parameters, the Monin-Obukhov (Monin and Obukhov 1954) theory is adopted. Simple log-linear similarity functions $\phi_{m, h}$ are chosen according to $\phi_{m, h}=1+\alpha z / L$ as a reasonable representative of atmospheric observations (Högström 1996). In VWD it is discussed that more complex similarity functions will generally lead to similar behavior. Also, in this latter study, effects of vertical flux divergence are formally accounted for. Here, we prefer a simple, vertically integrated version of the Monin-Obukhov theory. For an in-depth comparison between both approaches, we refer to VDW, Delage (1997), and Edwards (2009). As such fluxes are related to discrete wind and temperature differences over the bulk layer (Louis 1979),

$$
\begin{aligned}
H & =\rho c_{p} c_{D} U_{\text {cross }} \Delta T \cdot f\left(R_{b}\right) \\
\tau & =\rho c_{D} U_{\text {cross }}^{2} \cdot f\left(R_{b}\right),
\end{aligned}
$$

with the bulk Richardson number defined here as $R_{b} \equiv z_{\text {cross }}\left(g / \theta_{0}\right)\left(\Delta T / U_{\text {cross }}^{2}\right)$. The density of dry air $\rho$ is set to $1.2 \mathrm{~kg} \mathrm{~m}^{-3}, c_{p}$ is the heat capacity of air at constant pressure $\left(1005 \mathrm{~J} \mathrm{~kg}^{-1} \mathrm{~K}^{-1}\right), c_{D}$ is the neutral drag coefficient $c_{D}=\left[\kappa / \ln \left(z_{\text {cross }} / z_{0}\right)\right]^{2}, \kappa$ is the von Kármán constant (0.4), and $\theta_{0}$ is the reference temperature $(285 \mathrm{~K})$. By definition, $z_{\text {cross }}$ is taken at the $\mathrm{CP}$ (here, $40 \mathrm{~m}$ ), $z_{0}$ is the momentum roughness length $(0.1 \mathrm{~m})$, and $\tau$ is the turbulent stress. The aforementioned similarity functions $\phi_{m, h}$ can be converted in Richardson number form $f\left(R_{b}\right)$, leading to (e.g., England and McNider 1995; King and Connolly 1997)

$$
\begin{aligned}
& f\left(R_{b}\right)=\left(1-\alpha R_{b}\right)^{2} \text { for } R_{b} \leq 1 / \alpha \\
& f\left(R_{b}\right)=0 \text { for } R_{b}>1 / \alpha .
\end{aligned}
$$

Note that the impact of the stability function is nontrivial, in a sense that collapse is reached at $R_{b}$ values significantly lower than their "critical" value $1 / \alpha$. In fact, it can be shown that for $\lambda=0$, collapse already sets in at $R_{b}=1 / 3 \alpha$ (Van de Wiel et al. 2007). This corresponds to $z_{\text {cross }} / L$ values lower than 1 , so that the log-linear similarity functions are mainly used in the range for which they are supported by observations. After collapsethat is, when higher values of $z_{\text {cross }} / L$ occur-log-linear functions tend to lose validity: a hard critical Richardson number as implied by Eq. (5) does not seem to exist in real geophysical flows (Galperin et al. 2007). The predicted collapse of turbulence therefore merely suggests the appearance of a different, very stable regime, with turbulence levels that are of one order of magnitude lower than in the weakly stable regime (cf. Sun et al. 2012; Zilitinkevich et al. 2008; Mahrt 2011).

The full model combines Eqs. (1)-(5) to

$Q_{n}=\lambda \Delta T+\rho c_{p} c_{D} U_{\text {cross }} \Delta T\left[1-\alpha z_{\text {cross }}\left(g / \theta_{0}\right)\left(\Delta T / U_{\text {cross }}^{2}\right)\right]^{2}$

using the definition of the bulk Richardson number with $R_{b} \leq 1 / \alpha$. As before, all fluxes are defined positive. As $Q_{n}$ and $U_{\text {cross }}$ are assumed to be known, one unknown variable $\Delta T$ remains. Instead of considering $\Delta T$ as an unknown, one could equivalently consider the dimensionless $R_{b}$ to be the unknown. Below, this second option will be taken in order to facilitate interpretation.

\section{Results}

The terms in Eq. (6) are plotted as a function of $\alpha R_{b}$ for the case $Q_{n}=25 \mathrm{~W} \mathrm{~m}^{-2}$ and $\lambda=6$ in Fig. 3. Two cases are considered: $U_{\text {cross }}=7 \mathrm{~m} \mathrm{~s}^{-1}$ (Fig. 3a) and 
$U_{\text {cross }}=5 \mathrm{~m} \mathrm{~s}^{-1}$ (Fig. 3b). The left-hand side of Eq. (6) is represented by the gray line and the right-hand side by the black line. For the $7 \mathrm{~m} \mathrm{~s}^{-1}$ case, a balance is possible at the intersect $\alpha R_{b} \approx 0.18$ (black dot). Interestingly, no such intersect is found in case $U_{\text {cross }}=\mathrm{m} \mathrm{s}^{-1}$. Apparently, insufficient mechanical forcing is available in order to generate enough heat supply.

For completeness we mention that for exceptional cases with small $\lambda$ values, dual equilibria may occur. This aspect is discussed in detail by Van de Wiel et al. (2007), who show that in that case, the equilibrium point at large $\alpha R_{b}$ is unstable to perturbations under flux-driven boundary conditions. One may verify that the high- $\alpha R_{b}$ solution will be attracted to the low- $\alpha R_{b}$ solution in the case of small negative perturbation in $\alpha R_{b}$. For brevity reasons this aspect will not be elaborated and tacitly the stable, low- $\alpha R_{b}$ solution will be considered in the case of two equilibria. The most relevant point here is that the mere existence of any solution is highly sensitive to the ambient wind speed. Below, the consequences of this interesting feature are explored.

After solving Eq. (6) for $\Delta T$, the turbulent stress is diagnosed via Eq. (4). The procedure is repeated for various cases with different $U_{\text {cross }}$ values. In this way a relation between $\tau$ and $U_{\text {cross }}$ is obtained, which can be compared with the observations of Fig. 1. However, first, a few more observational characteristics are given. For the analysis a 10-yr dataset from a 200-m Cabauw tower is used. The tower is operated by the KNMI. The site is situated over fairly homogeneous grassland [for more site details, see Van Ulden and Wieringa (1996)]. To guarantee relatively stationary, cloud-free synoptic conditions, the following selection criteria are applied: only nights with less than $5 \mathrm{~m} \mathrm{~s}^{-1}$ variation of the geostrophic wind between 1200 and 0000 UTC and with continuously more than $20 \mathrm{~W} \mathrm{~m}^{-2}$ net radiative cooling are considered. The geostrophic wind was obtained by analyzing surface pressure observations from eight synoptic stations in a radius of $75 \mathrm{~km}$ around Cabauw (F. C. Bosveld 2012, personal communication). Turbulent fluxes were obtained using an eddy covariance technique, applied to $10-\mathrm{Hz}$ data from a sonic anemometer operated at $5 \mathrm{~m}$ above ground level. For each night the average stress and the average wind speed in the first $4 \mathrm{~h}$ after sunset were calculated and are represented by a dot marker.

In Fig. 4 the modeled relation between $\tau$ and $U_{\text {cross }}$ is compared to the observations of Fig. 1. For completeness we note that the minimum wind speed that allows a balance in Eq. (6) corresponds to a small but finite value of $\tau$. According to Eq. (6) smaller wind speeds do not allow a steady flux balance in Eq. (6), so that nearsurface stability will rapidly increase and $\tau$ tends to zero values (hence, the interpolation between the minimum

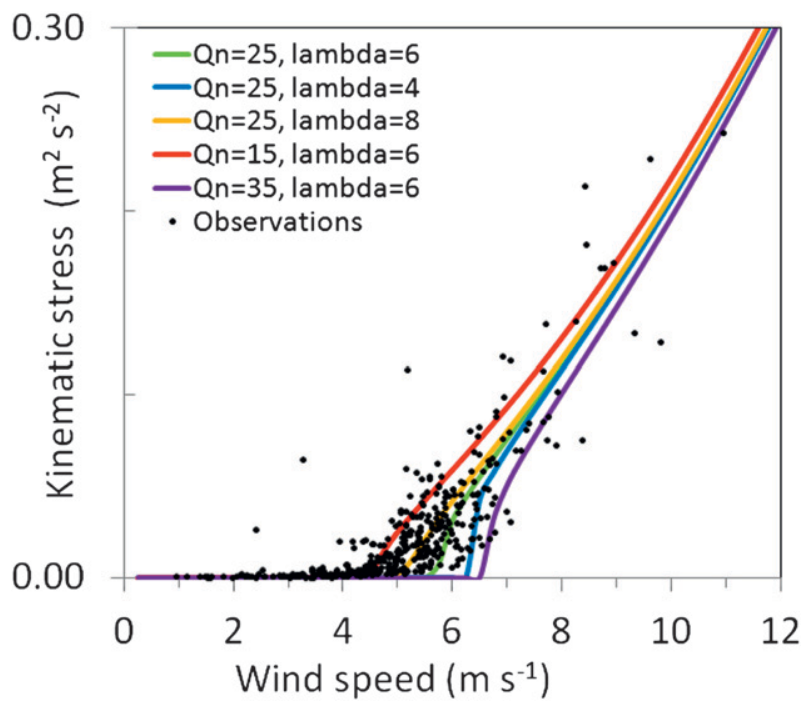

FIG. 4. Nighttime kinematic turbulent stress (at $5 \mathrm{~m}$ ) as a function of the wind speed at $40 \mathrm{~m}$ for clear-sky situations observed at the KNMI Cabauw observatory (dots). For comparison, model predictions [Eqs. (6) and (4)] are given for various classes of net radiative cooling $Q_{n}$ and for various values of the soil-vegetation conductivity parameter $\lambda$. Observe that the predicted shape of the relation between stress and wind forcing is rather robust to the parameter changes indicated.

stress value and zero is made in Fig. 4). Model results are given for a range of $Q_{n}$ and $\lambda$, rather than by presentation of a single line. This is to emphasize that the model results are intended as proof of principle only. A really detailed prediction would require accurate knowledge on model parameters, which is often not available. Nevertheless, the robustness of the predicted relation between $\tau$ and $U_{\text {cross }}$ is remarkable. The reason for this is explained in the next section. Generally speaking, the typical shape of the observational data cluster appears to be captured by the model. Observe that for cases of strong radiative cooling, higher wind speeds are needed in order to sustain turbulence, as is to be expected. Cases with small $\lambda$ values correspond to surfaces with strongly isolating properties. Those properties, again, favor strong surface cooling and likewise higher speeds are needed to sustain turbulence.

\section{Interpretation}

In this section the reason for the model robustness is explained. To this end Eq. (3) is rewritten using Eq. (5) as

$$
H \cdot \frac{g}{\theta_{0}} \frac{\alpha z_{\text {cross }}}{\rho c_{p} c_{D} U_{\text {cross }}^{3}}=\alpha R_{b}\left(1-\alpha R_{b}\right)^{2} .
$$

By differentiating Eq. (7) with respect to $\alpha R_{b}$, an expression for the maximum achievable heat flux is obtained. 
The maximum is reached at moderate stability, $\alpha R_{b}=1 / 3$, and reads (Taylor 1971; Malhi 1995)

$$
H_{\max }=\frac{4}{27} \frac{\kappa^{2}}{\alpha} \frac{\rho c_{p} \theta_{0}}{g} \frac{U_{\text {cross }}^{3}}{z_{\text {cross }} \cdot\left[\ln \left(z_{\text {cross }} / z_{0}\right)\right]^{2}}
$$

using the definition of $c_{D}$. It occurs that the maximum is proportional to the cube of $U_{\text {cross }}$ [cf. Fig. 3 of the previous section and Fig. 5 in Delage et al. (2002)]. It is therefore very sensitive to this forcing. For interpretation purposes let us assume that $G$ is a priori given. Then, the minimum wind speed for sustained turbulence is found directly by substituting $H=H_{\max }$ in Eq. (1), giving

$$
U_{\min }=\left\{\frac{27}{4} \frac{\alpha g}{\theta_{0} \kappa^{2}} \frac{\left|Q_{n}\right|-|G|}{\rho c_{p}} \cdot z_{\text {cross }}\left[\ln \left(z_{\text {cross }} / z_{0}\right)\right]^{2}\right\}^{1 / 3} .
$$

It becomes clear why the minimum wind speed in Fig. 4 is rather insensitive to the exact value of $Q_{n}$ and $\lambda$ : it is the cubic dependence in Eq. (8) that causes the minimum wind speed to be only weakly dependent on the exact energy demand $\left|Q_{n}\right|-|G|$ in Eq. (9). In that perspective, our crude parameterization in Eq. (2) appears to be justified.

From this fact, it is possible to anticipate a cessation of continuous turbulence of strongly isolated surfaces, such as fresh snow, in the case of weak winds and clear skies. Let us, for example, assume a radiative heat loss of $40 \mathrm{~W} \mathrm{~m}^{-2}$. For $\lambda$ we assume a value of $5 \mathrm{~W} \mathrm{~m}^{-2} \mathrm{~K}^{-1}$, comparable to the value for short grass. Without further knowledge $z_{\text {cross }}$ is set to $40 \mathrm{~m}$ and the roughness length to $0.01 \mathrm{~m}$. We also assume that the wind at crossing height is unknown, but that an estimate of the geostrophic wind is available. In that case, we may use the fact that the actual geostrophic wind $U_{\text {geo }}$ is generally larger than the wind at crossing height and hence it can be considered as an upper bound for the latter. Next, the consequence of a low mechanical forcing, say, $U_{\text {geo }}=4 \mathrm{~m} \mathrm{~s}^{-1}$, for the expected intensity of turbulence is evaluated. From Eq. (8) the achievable $H_{\max }$ will be at most $4 \mathrm{~W} \mathrm{~m}^{-2}$, that is, less than $10 \%$ of the net radiative heat loss. To reach a balance at the surface, the soil heat flux must amount to $36 \mathrm{~W} \mathrm{~m}^{-2}$, so that the expected inversion strength $\Delta T$ is at least $7 \mathrm{~K}$ [Eq. (2)]. The corresponding $R_{b}$ will then be larger than 0.6 , that is, 3 times higher than the "critical" value of 0.2 [Eq. (5)], so that sustained turbulence is unlikely in this case. In Table 1 the calculation is repeated for several values of $U_{\text {geo }}$, and it occurs that subcritical Richardson numbers, that is, in terms of Eq. (5), become possible only for $U_{\text {geo }}>6 \mathrm{~m} \mathrm{~s}^{-1}$.
TABLE 1. Maximum sustainable heat flux in response to wind forcing according to Eq. (8), with $U_{\text {geo }}$ as an upper estimate for $U_{\text {cross }}$, the wind speed at crossing height. This example considers a strongly isolated surface with a net radiation of $40 \mathrm{~W} \mathrm{~m}^{-2}$ (see text). Consequences are given in case $H_{\max }$ were to be reached for the soil heat flux, the inversion strength, and the bulk Richardson number. Only for $U_{\text {geo }}=7.5$ and $U_{\text {geo }}=8.5 \mathrm{~m} \mathrm{~s}^{-1}$ "subcritical" Richardson numbers are possible in order to sustain significant, continuous turbulence.

\begin{tabular}{ccccc}
\hline \hline$U_{\text {geo }}\left(\mathrm{m} \mathrm{s}^{-1}\right)$ & $H_{\max }\left(\mathrm{W} \mathrm{m}^{-2}\right)$ & $G\left(\mathrm{~W} \mathrm{~m}^{-2}\right)$ & $\Delta T(\mathrm{~K})$ & $R_{b}$ \\
\hline 3 & 1.6 & 38.4 & 7.7 & 1.2 \\
4 & 3.9 & 36.1 & 7.2 & 0.6 \\
5 & 7.5 & 32.5 & 6.4 & 0.4 \\
6 & 13.0 & 27.0 & 5.4 & 0.2 \\
7.5 & 25.5 & 14.5 & 2.9 & 0.07 \\
8.5 & 37.1 & 2.9 & 0.6 & 0.01 \\
\hline
\end{tabular}

Apart from the interpretation issues, expression (9) also seems to provide a plausible explanation for the observational findings by Sun et al. (2012), as mentioned in the introduction. According to their results, the minimum wind speed for sustainable turbulence during the CASES-99 campaign is approximately logarithmically dependent on the height of the wind observation. Equation (9) supports such dependence. However, it is realized that the "choice" of the height of the wind observation is, strictly speaking, not "free" in our model: our analysis formally only applies to the wind speed at crossing level.

\section{The minimum geostrophic wind speed for sustainable turbulence}

The classification into strongly turbulent and weakly turbulent evening boundary layers in Fig. 4 was constructed by using specific information on the magnitude of the wind speed at crossing level. Information about such an internal variable is usually not available in practice. As shown by Nieuwstadt (2005), Flores and Riley (2011), and VDW, the response of the nocturnal boundary layer as a whole depends critically on external forcing conditions. The mechanical forcing comes from the synoptic pressure gradient, or, in the case of barotropic conditions, from the geostrophic wind. The buoyancy is imposed via prescribed $Q_{n}$. As discussed in section 2, $Q_{n}$ can be considered as a pseudoexternal parameter, as its magnitude primarily depends on the presence/absence of low-level clouds, and to a lesser extent on the surface cooling itself (the decrease in outgoing radiative loss is largely compensated by the decreasing incoming radiation due to BL cooling). Next, two cases are considered:

1) prescribed $Q_{n}$ and

2) prescribed $Q_{n}-G$ (or, equivalently, prescribed $H_{0}$ ). 


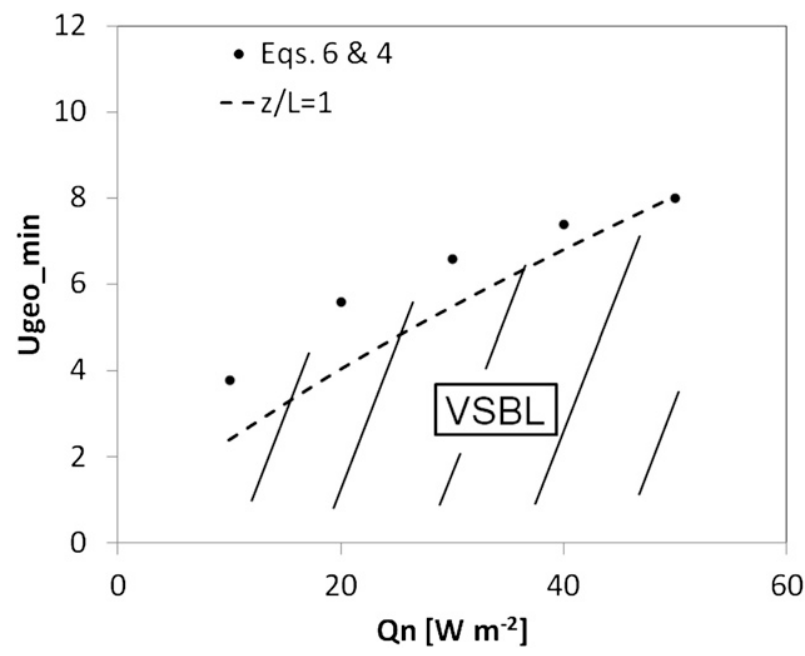

FIG. 5. Minimum geostrophic wind speed for sustainable continuous turbulence for an ABL forced by a constant net radiation at the surface (black dots). By substituting the generally larger $U_{\text {geo }}$ for $U_{\text {cross }}$ in Eqs. (6) and (4), a "safe" estimate for the critical $U_{\text {geo }}$ is obtained (see text). Below this threshold, the onset of the VSBL is foreseen (shaded area). The dashed line (with $z=10 \mathrm{~m}$ ) indicates favorable conditions for the emergence of the VSBL according the $z / L=1$ criterion given by Mahrt et al. $(1998 ; z=10)$. The translation from $z / L=1$ to $U_{\text {geo }}-Q_{n}$ space in the plot is made using the model of Donda et al. (2012). Here, for reasons of consistency, both models assume that $\lambda=5 \mathrm{~W} \mathrm{~m}^{-2} \mathrm{~K}^{-1}$.

\section{a. Case 1}

This case is the most realistic case, as it keeps the turbulent heat flux and the soil heat flux as dependent internal variables and predictions can be compared directly to observations. Here, a regime classification in terms of $U_{\text {geo }}$ and $Q_{n}$ is made by taking Fig. 4 as the point of departure. In that graph the required wind speed for sustainable turbulence increases with increasing $Q_{n}$ values (red, green, purple lines). Next, we use the fact that $U_{\text {cross }}<U_{\text {geo }}$ : if $U_{\text {cross }}$ is insufficient to sustain turbulence, then $U_{\text {geo }}$ certainly is. As such the threshold values for $U_{\text {cross }}$ in Fig. 4 can be interpreted as a lower bound for the critical $U_{\text {geo }}$ itself. Figure 5 depicts the critical $U_{\text {geo }}$ as a function of $Q_{n}$ (black dots). For reasons of consistency with Donda et al. (2012; see below) here $\lambda=5 \mathrm{~W} \mathrm{~m}^{-2} \mathrm{~K}^{-1}$ is taken, but by combining Figs. 4 and 5 , it is readily verified that $\lambda=6 \mathrm{~W} \mathrm{~m}^{-2} \mathrm{~K}^{-1}$ leads to comparable results. The critical $U_{\text {geo }}$ typically ranges between 5 and $8 \mathrm{~m} \mathrm{~s}^{-1}$, for $Q_{n}>20 \mathrm{~W} \mathrm{~m}{ }^{-2}$. This conclusion seems to be supported by observations from the Cabauw tower in VDW, where it is shown that under clear-sky conditions, turbulent stresses become small for $U_{\text {geo }}=6 \pm 1 \mathrm{~m} \mathrm{~s}^{-1}$ and have negligible values in the interval $U_{\text {geo }}=3 \pm 1 \mathrm{~m} \mathrm{~s}^{-1}$. Specific observational research on this topic is needed for a more precise determination of the critical geostrophic wind at this location.

In Mahrt et al. (1998) nocturnal boundary layers are classified according to their turbulence characteristics in relation to the stability parameter $z / L$ as observed at 10 -m height. They showed that near-surface turbulence characteristics start to change rapidly from $z / L \approx 0.1$. Then, beyond $z / L \approx 1$ a new regime has been established with turbulence characteristics largely different from the near-neutral case. This regime is referred to as the very stable boundary layer [see also Smedman (1988)]. Of course, $z / L \approx 1$ cannot serve as a hard, universal criterion for the appearance of the VSBL, because the choice of the observational height $z$ is arbitrary [this was already recognized by Mahrt et al. (1998) and, e.g., by Derbyshire (1999b)]. In contrast, by taking a fixed observational level by convention, say, $10 \mathrm{~m}$, a one-to-one relation between the stability at this height and the external parameters is established, so that the regime transition corresponds to a certain critical value of $z / L$. In that perspective the success of classifications in terms of $z / L$ can be understood. It is therefore tempting to translate the $z / L=1$ criterion in terms of external parameters for the Cabauw case. Hereto recent findings by Donda et al. (2012) are used, who developed a model in order to relate internal parameters such as $u_{*}, \theta_{*}$, and $L$ to $U_{\text {geo }}$ and $Q_{n}$ (see the appendix). From this they used the Monin-Obukov similarity theory to predict nearsurface wind and temperature profiles. Predictions were validated against a dataset covering 11 yr of observations and a close agreement was found. The model is used here to predict the $z / L=1$ line in terms of $U_{\text {geo }}$ and $Q_{n}$. The result is given by the dashed line in Fig. 5 . Though the methodology is clearly too qualitative and indirect to justify rigorous conclusions, it appears that both classifications are broadly in accordance with respect to the parameter regime where the emergence of the VSBL can be foreseen.

In connection with this, the parameter $h / L$ has also been proposed as a diagnostic of nocturnal boundary layer regimes (e.g., Holtslag and Nieuwstadt 1986), with $h$ as the height of the turbulent boundary layer. As with $z / L$, such a parameter has proven to be useful in order to stratify observational data into subcategories. The major disadvantage is, again, the use of $L$ as an internal parameter. The same is true for $h$, a parameter that is not always unambiguous to determine from in situ observations (Beyrich and Weill 1993). An exception to those statements occurs in case of flux-driven, stratified channel flows as presented in Nieuwstadt (2005), Flores and Riley (2011), and VDW. In that specific case, the equilibrium value of $h / L$ can be expressed analytically in terms of the pressure gradient and the surface heat flux. 


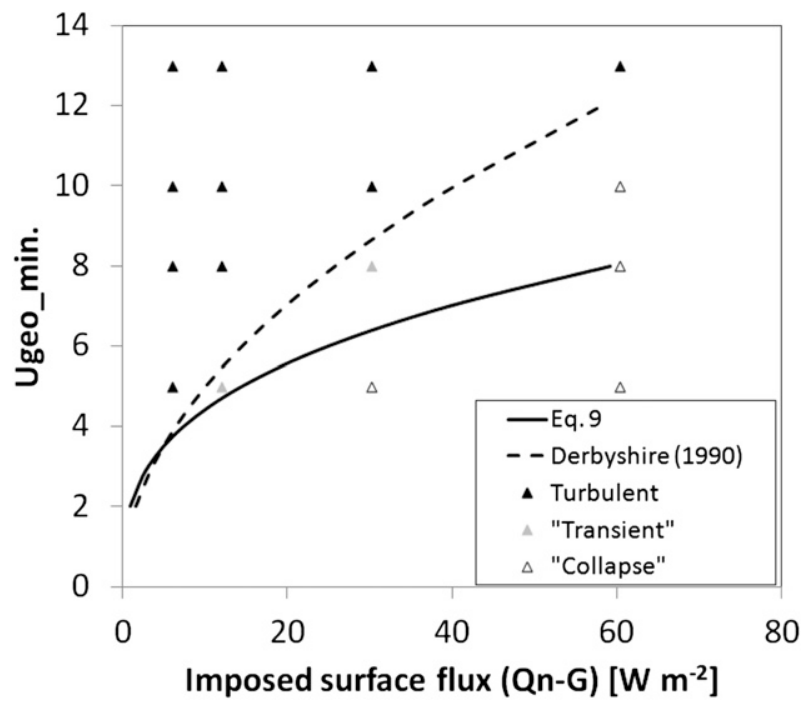

FIG. 6. Minimum geostrophic wind speed for sustainable turbulence in case of an imposed surface heat flux $H_{0}=Q_{n}-G$ (all defined positive). Results of Jiménez and Cuxart (2005) are given by symbols. From LESs they diagnosed: continuous turbulent SBLs (black triangles), SBLs with collapsed turbulence (open/ white triangles), and transient cases (gray triangles). Note that Zhou and Chow (2011) argue that the simulations by Jiménez and Cuxart may have an overtendency to collapse due to enhanced dissipation by their subgrid scheme (see text). For comparison, the maximum heat flux that can be converted from the work by pressure gradient is given, for the case in which the maximum bulk Richardson number $R_{f C}$ equals 0.25 (Derbyshire 1990).

\section{b. Case 2}

\section{A PRESCRIBED SURFACE HEAT FLUX}

For this case there is no need to parameterize the effect of soil heat transport, and therefore Eq. (9) can be used directly to infer the minimum wind speed. As before, we diagnose the minimum $U_{\text {geo }}$ instead of the minimum $U_{\text {cross }}$. We take $z_{\text {cross }}=40 \mathrm{~m}$ and assume the roughness length of $0.1 \mathrm{~m}$ for consistency with results by Jiménez and Cuxart (2005), which will be compared. By using large-eddy simulations, they investigate the response of SBL turbulence characteristics to external forcings. For each case they diagnosed if the model was able to sustain well-developed, continuous turbulence. In Fig. 6 those cases are depicted by the full black triangles. For some cases runaway cooling occurred (open triangles), whereas other cases had an "in between," transient character (gray triangles). The predictions by Eq. (9) are given by the full line. We also plotted the surface heat flux that follows from Nieuwstadt's (1985) model in the limit of $h / L \rightarrow \infty$ (Derbyshire 1990) as

$$
H=\rho c_{p} \frac{\theta_{0}}{g} \frac{R_{f}}{\sqrt{3}} f_{c} U_{\mathrm{geo}}^{2} .
$$

Nieuwstadt's model assumes that Ri and $R_{f}$ are constant throughout the boundary layer. The graph represents the case $R_{f} \cong 0.25$. According to Derbyshire we may interpret Eq. (10) also as an upper bound to the surface heat flux, that is, $H=H_{\max }$, as it also represents an energy constraint: the global mixing efficiency $R_{f}$ of stably stratified turbulence is normally less than around 0.25 , and certainly less than 1 in any quasi-steady problem. In this rationale, the maximum sustainable heat flux must be proportional to this maximum "multiplied" by the kinetic energy production, which ultimately is generated by the work done by the pressure gradient (cf. Wyngaard 2010 , p. 285). Recently, this picture was refined by Zilitinkevich et al. (2008), who use the concept of total turbulent energy to show that the critical $R_{f}$ separates strongly turbulent states from weakly turbulent states rather than from truly laminar states.

Even more importantly, Eq. (10) is inferred from boundary layer scaling alone, whereas more recent work by Derbyshire (1999b,a) has proven that the hydrodynamic stability of a system as a whole cannot be viewed apart from its (surface) boundary conditions. He states in Derbyshire (1999a, p. 299): "So even the simplest valid analysis needs to couple explicitly the wind profile, temperature profile and surface heat budget," and in Derbyshire (1999b, p. 431) he states: "Hence the SBL is one case where we may not be able to separate boundary layer behaviour from boundary condition specification." It fact, those statements are founded in classical theory on hydrodynamic stability (Drazin and Reid 2004). For example, the critical Rayleigh number $\mathrm{Ra}_{C}$ for the onset of convection in response to fluid heating between two rigid plates is reduced by more than $50 \%$ by changing to stressfree boundary conditions in the same system (Kundu and Cohen 2008). From this perspective Eq. (10) in Fig. 6 should be viewed as some point of reference rather than a true predictor of WSBL instability as aimed for by Eq. (9).

Nevertheless, it is surprising how well the $R_{f} \cong 0.25$ criterion represented by Eq. (10) separates the various regimes encountered by Jiménez and Cuxart (2005). In contrast, Zhou and Chow (2011) argue that the original Smagorinsky subgrid closure scheme used in the LESs by Jiménez and Cuxart (2005) is too dissipative and hence will too easily favor flow laminarization. They propose an improved scheme-the dynamic reconstruction model-and showed that turbulence sustained for the $U_{\text {geo }}=10 \mathrm{~m} \mathrm{~s}^{-1}$ case that collapsed in Jiménez and Cuxart (2005). Unfortunately, they did not repeat the lowwind, numerical experiments by Jiménez and Cuxart with the original flux boundary condition. Instead, they used prescribed temperature boundary conditions for the higher-stability classes. Below, it is argued that the latter condition effectively suppresses runaway cooling. 
We summarize Figs. 4-6 by stating that under clearsky conditions over short grass, sustained and continuous turbulence is unlikely to occur when the geostrophic wind is below about $5 \mathrm{~m} \mathrm{~s}^{-1}$ in magnitude.

\section{Impact of surface boundary conditions}

In our prototype system, the storage term in the surface energy balance [Eq. (1)] was neglected (Van de Wiel et al. 2002a). As such, the analysis is an approximation for surfaces with relatively small heat capacity. It is well known that large surface heat capacity (e.g., with water surfaces) may have a strongly moderating effect on the collapse phenomenon, as it effectively prevents rapid surface cooling (Revelle 1993; McNider et al. 1995; Derbyshire 1999a; Delage et al. 2002; Van de Wiel et al. 2002a). In its present form, cooling of the model is driven by $Q_{n}$. Our system is therefore basically governed by a flux boundary condition. Clearly, such system behaves differently from a temperature-driven boundary layer that may, for example, evolve over melting snow. In the latter case, runaway cooling of the lower atmosphere is naturally prevented by the fixed temperature boundary condition itself. An illustration of this is given by the weak wind simulations presented in Kosović and Curry (2000). In theory, a weak-wind, flux-driven case and the temperature-driven case may even lead to identical equilibrium profiles for wind and temperature, while having totally different hydrodynamic stability [of course, for high winds they will both be hydrodynamically stable as, e.g., in Fig. 5 in Jiménez and Cuxart (2005)]. Turbulence could cease over dry snow while the melting snow case could be in equilibrium under similar external forcings. The flux-driven view to the collapse mechanism in itself is not an artifact, as it corresponds to a real situation (Wyngaard 2010, p. 285). However, for realistic numerical modeling of collapse one should avoid prescribing either surface fluxes or temperatures directly. As shown in Basu et al. (2008), the dual character of the turbulent heat flux will lead to problems in simulating high stabilities that occur after collapse (not modeled here). Likewise, correct time dependence of surface temperature in relation to large-scale forcings cannot be known in advance (Holtslag et al. 2007), and prescribed surface temperatures limit the dynamic freedom of the system. Therefore, usage of a coupled atmosphere-land surface model will be the most realistic as it includes both asymptotic cases.

\section{Conclusions}

A common feature in evening boundary layers is the appearance of a so-called crossing point in the velocity profile. At this level, typically around $30-60 \mathrm{~m}$, wind speeds have a tendency to remain relatively "stationary" in magnitude, that is, as compared to high-level winds that usually show significant accelerations due to inertial effects and compared to near-surface winds that tend to weaken in the evening (VDW). Observations from Cabauw indicate that a minimum wind speed at crossing height is needed in order to maintain continuous turbulence of significant magnitude, in accordance with results for CASES-99 data given by Sun et al. (2012). Here, a simple atmospheric bulk model is used to explain the relation between the wind speed at crossing height and the intensity of turbulence in the evening boundary layer. The model incorporates the fact that for a given wind speed, the maximum amount of heat that can be transported to the surface is limited to a certain maximum. In case the energy demand at the surface, basically the net radiation minus the soil heat transport, is significantly larger than this turbulent heat supply, the near-surface inversion will rapidly intensify (i.e., if we leave heat storage processes out of consideration, as in the present study). This causes turbulent heat transport to be reduced further, so that eventually turbulent activity is largely suppressed. Although the exact value of the critical speed depends on the physical properties of the surface, it is shown that for clear skies, the threshold is usually close to $5-7 \mathrm{~m} \mathrm{~s}^{-1}$. As the geostrophic wind speed usually is larger than the speed at crossing height, $5 \mathrm{~m} \mathrm{~s}^{-1}$ can also be considered as a lower bound for the critical geostrophic wind needed to allow weakly stable, continuous turbulent boundary layers.

In any case it is realized that the current model setup still relies on rather crude assumptions and simplifications. Therefore, this research topic will largely benefit from comparison with more advanced modeling and comparison with observations from various locations, so that particularly the role of surface boundary conditions in relation to regime transitions can be further clarified.

Acknowledgments. We are thankful to the Royal Netherlands Meteorological Institute (KNMI), in particular to Dr. Fred Bosveld, for having provided the observational data presented in Figs. 1 and 4. The research has been supported by a VIDI grant (10011425) from the Dutch National Science Foundation (NWO), which is gratefully acknowledged.

\section{APPENDIX}

\section{Predicting Nocturnal Wind and Temperature Profiles Based on External Forcing Parameters}

In section $5, z / L=1$ (with $z=10 \mathrm{~m}$ ) as indicator of favorable WSBL conditions is translated in terms of $U_{\text {geo }}$ 
and $Q_{n}$ utilizing the work of Donda et al. (2012). A brief summary is given here. For more details, refer to the original paper. By coupling an Ekman model to a rudimentary surface energy balance, a scaling framework results, which expresses surface fluxes in terms of "external" parameters $U_{\text {geo }}$ and $Q_{n}$. Next, two calibration constants (one for heat and one for momentum) are fixed by comparing the scaling with numerical simulations of a $1 \mathrm{D}$ atmospheresurface model in the range of $5<U_{\text {geo }}<15 \mathrm{~m} \mathrm{~s}^{-1}$. As such, the following expressions are found:

$$
u_{*}=2.34 \frac{\theta_{0} f_{c} \lambda}{g_{\kappa}} \frac{U_{\mathrm{geo}}^{2}}{Q_{n}} \quad \text { and } \quad \theta_{*}=0.11 \frac{\kappa}{\alpha \lambda} Q_{n},
$$

with $f_{c}$ as the Coriolis parameter $\left(10^{-4} \mathrm{~s}^{-1}\right)$ and other symbols as defined in the present text. The model assumes validity of the Monin-Obukhov similarity theory in order to predict near-surface wind and temperature profiles up to $z=L$. The predictions were validated against an independent dataset that covers $11 \mathrm{yr}$ of observations at Cabauw. The characteristic climatology, which also closely compares with European Centre for Medium-Range Weather Forecasts (ECMWF) reanalysis data, is well captured by the conceptual model. We therefore assume that a reasonably realistic estimate of $u_{*}$ and $\theta_{*}$ in terms of external parameters is made by following this work. Finally, we use the definition $L \equiv\left(\theta_{0} / \kappa g\right)\left(u_{*}^{2} / \theta_{*}\right)$ to calculate the $z / L=1$ line in Fig. 5 at $z=10 \mathrm{~m}$. Though the present analysis is meant as a rudimentary exercise only, it must be noted that the region $U_{\text {geo }}=5 \mathrm{~m} \mathrm{~s}^{-1}$ in Fig. 5 formally falls outside the calibration range of the aforementioned constants.

\section{REFERENCES}

Acevedo, O. C., F. D. Costa, and G. A. Degrazia, 2012: The coupling state of an idealized stable boundary layer. Bound.Layer Meteor., 145, 211-228, doi:10.1007/s10546-011-9676-3.

Baas, P., B. J. H. Van de Wiel, L. van den Brink, and A. A. M. Holtslag, 2012: Composite hodographs and inertial oscillations in the nocturnal boundary layer. Quart. J. Roy. Meteor. Soc., 138, 528-535, doi:10.1002/qj.941.

Baklanov, and Coauthors, 2011: The nature, theory, and modeling of atmospheric planetary boundary layers. Bull. Amer. Meteor. Soc., 92, 123-128.

Banta, R. M., 2008: Stable boundary-layer regimes from the perspective of the low-level jet. Acta Geophys., 56, 58-87.

Basu, S., F. Porté-Agel, E. Foufoula-Georgiou, J.-F. Vinuesa, and M. Pahlow, 2006: Revisiting the local scaling hypothesis in stably stratified atmospheric boundary layer turbulence: An integration of field and laboratory measurements with large-eddy simulations. Bound.-Layer Meteor., 119, 473-500.

, A. A. M. Holtslag, B. J. H. Van de Wiel, A. F. Moene, and G.-J. Steeneveld, 2008: An inconvenient "truth" about using sensible heat flux as a surface boundary condition in models under stably stratified regimes. Acta Geophys., 56, 88-99.

Beare, R. J., and Coauthors, 2006: An intercomparison of largeeddy simulations of the stable boundary layer. Bound.-Layer Meteor., 118, 247-272.

Beyrich, F., and A. Weill, 1993: Some aspects of determining the stable boundary layer depth from sodar data. Bound.-Layer Meteor., 63, 97-116.

Blackadar, A. K., 1957: Boundary layer wind maxima and their significance for the growth of nocturnal inversions. Bull. Amer. Meteor. Soc., 38, 283-290.

Brunt, P., 1941: Physical and Dynamical Meteorology. Cambridge University Press, 428 pp.

Businger, J. A., 1973: Turbulent transfer in the atmospheric surface layer. Workshop on Micrometeorology, D. A. Haugen, Ed., Amer. Meteor. Soc., 67-98.

Cuxart, J., and Coauthors, 2006: Single-column model intercomparison for a stably stratified atmospheric boundary layer. Bound.-Layer Meteor., 118, 427-432.

De Bruin, H. A. R., 1994: Analytic solutions of the equations governing the temperature fluctuation method. Bound.-Layer Meteor., 68, 427-432.

Delage, Y., 1997: Parameterising sub-grid scale vertical transport in atmospheric models under statistically stable conditions. Bound.-Layer Meteor., 82, 23-48.

— , P. A. Bartlett, and J. H. McCaughey, 2002: Study of 'soft' night-time surface-layer decoupling over forest canopies in a land-surface model. Bound.-Layer Meteor., 103, 253-276.

Derbyshire, S. H., 1990: Nieuwstadt's stable boundary layer revisited. Quart. J. Roy. Meteor. Soc., 116, 127-158.

_ 1999a: Boundary-layer decoupling over cold surfaces as a physical boundary instability. Bound.-Layer Meteor., 90, 297-325.

_ 1999 b: Stable boundary layer modeling: Established approaches and beyond. Bound.-Layer Meteor., 90, 423-446.

Donda, J. M. M., B. J. H. Van de Wiel, F. C. Bosveld, F. Beyrich, G. J. F. van Heijst, and H. J. H. Clercx, 2012: Predicting nocturnal wind and temperature profiles based on external forcing parameters. Bound.-Layer Meteor., doi:10.1007/s10546-0129755-0, in press.

Drazin, P. G., and W. H. Reid, 2004: Hydrodynamic Stability. Cambridge University Press, 605 pp.

Duynkerke, P. G., 1999: Turbulence, radiation and fog in Dutch stable boundary layer. Bound.-Layer Meteor., 90, 447-477.

Edwards, J. M., 2009: Radiative processes in the stable boundary layer: Part II. The development of the nocturnal boundary layer. Bound.-Layer Meteor., 131, 127-146.

England, D. E., and R. T. McNider, 1995: Stability functions based upon shear functions. Bound.-Layer Meteor., 74, 113-130.

Estournel, C., and D. Guedalia, 1985: Influence of geostrophic wind on atmospheric nocturnal cooling. J. Atmos. Sci., 42, 2695-2700.

Fernando, H. J. S., and J. C. Weil, 2010: Whither the stable boundary layer? Bull. Amer. Meteor. Soc., 91, 1475-1484.

Flores, O., and J. J. Riley, 2011: Analysis of turbulence collapse in the stably stratified surface layer using direct numerical simulation. Bound.-Layer Meteor., 139, 241-259.

Galperin, B., S. Sukoriansky, and P. S. Anderson, 2007: On the critical Richardson number in stably stratified turbulence. Atmos. Sci. Lett., 8, 65-69.

Heusinkveld, B. G., A. F. G. Jacobs, A. A. M. Holtslag, and S. M. Berkowicz, 2004: Surface energy balance closure in an arid region: Role of soil and heat flux. Agric. For. Meteor., 122, 21-37. 
Högström, U., 1996: Review of some basic characteristics of the atmospheric surface layer. Bound.-Layer Meteor., 78, 215-246.

Holtslag, A. A. M., and F. T. M. Nieuwstadt, 1986: Scaling the atmospheric boundary layer. Bound.-Layer Meteor., 36, 201-209.

_ , and H. A. R. De Bruin, 1988: Applied modeling of the nighttime surface energy balance over land. J. Appl. Meteor., 27, 689-704.

_ - G.-J. Steeneveld, and B. J. H. Van de Wiel, 2007: Role of land-surface temperature feedback on model performance for the stable boundary layer. Bound.-Layer Meteor., 118, 103-116.

Jiménez, M. A., and J. Cuxart, 2005: Large-eddy simulations of the stable boundary layer using the standard Kolmogorov theory: Range of applicability. Bound.-Layer Meteor., 115, 241-261.

King, J. C., and W. M. Connolly, 1997: Validation of the surface energy balance over the Antarctic Ice Sheets in the U.K. Meteorological Office Unified Climate Model. J. Climate, 10, 1273-1287.

Kosović, B., and J. A. Curry, 2000: A large eddy simulation study of a quasi-steady, stably stratified atmospheric boundary layer. J. Atmos. Sci., 57, 11 052-11 068.

Kundu, P. K., and I. M. Cohen, 2008: Fluid Mechanics. 4th ed. Academic Press, 872 pp.

Lapworth, A., 2008: The evening wind. Weather, 63, 12-14.

Louis, J.-F., 1979: A parametric model of vertical eddy fluxes in the atmosphere. Bound.-Layer Meteor., 17, 187-202.

Mahrt, L., 2011: The near-calm stable boundary layer. Bound.Layer Meteor., 140, 343-360, doi:10.1007/s10546-011-9616-2.

_- J. Sun, W. Blumen, T. Delany, and S. Oncley, 1998: Nocturnal boundary layer regimes. Bound.-Layer Meteor., 88, 255-278.

Malhi, Y. S., 1995: The significance of the dual solutions for heat fluxes measured by the temperature fluctuation method in stable conditions. Bound.-Layer Meteor., 74, 389-396.

Mauritsen, T., and G. Svensson, 2007: Observations of stably stratified shear-driven atmospheric turbulence at low and high Richardson numbers. J. Atmos. Sci., 64, 645-655.

McNider, R. T., D. E. England, M. J. Friedman, and X. Shi, 1995: Predictability of the stable atmospheric boundary layer. J. Atmos. Sci., 52, 1602-1614.

Monin, A. S., and A. M. Obukhov, 1954: Basic laws of turbulent mixing in the atmosphere near the ground. Tr. Geofiz. Inst., Akad. Nauk SSSR, 24, 1963-1987.

Monteith, J. L., 1981: Evaporation and surface temperature. Quart. J. Roy. Meteor. Soc., 107, 1-27.

Nieuwstadt, F. T. M., 1984: The turbulent structure of the stable, nocturnal boundary layer. J. Atmos. Sci., 41, 2202-2216. 1985: A model for the stationary, stable boundary layer. Turbulence and Diffusion in Stable Environments: Based on the Proceedings of a Conference on Models of Turbulence and Diffusion in Stably Stratified Regions of the Natural Environment, J. C. R. Hunt, Ed., Oxford University Press, 149-179.

_ 2005: Direct numerical simulation of stable channel flow at large stability. Bound.-Layer Meteor., 116, 277-299.

Ohya, Y., D. E. Neff, and R. N. Merony, 1997: Turbulence structure in a stratified boundary layer under stable conditions. Bound.-Layer Meteor., 83, 139-161.

Poulos, G. S., and Coauthors, 2002: CASES-99: A comprehensive investigation of the stable nocturnal boundary layer. Bull. Amer. Meteor. Soc., 83, 555-581.

Revelle, D. O., 1993: Chaos and "bursting" in the planetary boundary layer. J. Appl. Meteor., 32, 1169-1180.

Schubert, J. F., 1977: Acoustic detection of momentum transfer during the abrupt transition from a laminar to a turbulent atmospheric boundary layer. J. Atmos. Sci., 16, 1292-1297.
Shapiro, A., and E. Fedorovich, 2010: Analytical description of a nocturnal low-level jet. Quart. J. Roy. Meteor. Soc., 136, $1255-1262$.

Smedman, A.-S., 1988: Observations of a multi-level turbulence structure in a very stable atmospheric boundary layer. Bound.Layer Meteor., 44, 231-253.

Sorbjan, Z., 2006: Local structure of turbulence in stably stratified boundary layers. J. Atmos. Sci., 63, 1526-1537.

Steeneveld, G. J., B. J. H. Van de Wiel, and A. A. M. Holtslag, 2006: Modeling the evolution of the atmospheric boundary layer coupled to the land surface for three contrasting nights in CASES-99. J. Atmos. Sci., 63, 920-935.

Sun, J., and Coauthors, 2003: Atmospheric disturbances that generate intermittent turbulence in nocturnal boundary layer. Bound.-Layer Meteor., 110, 255-279.

—, L. Mahrt, R. M. Banta, and Y. L. Pichugina, 2012: Turbulence regimes and turbulence intermittency in the stable boundary layer during CASES-99. J. Atmos. Sci., 69, 338-351.

Svensson, G., and Coauthors, 2011: Evaluation of the diurnal cycle in the atmospheric boundary layer over land as represented by a variety of single-column models: The second GABLS experiment. Bound.-Layer Meteor., 140, 177-206.

Taylor, P. A., 1971: A note on the log-linear velocity profile in stable conditions. Quart. J. Roy. Meteor. Soc., 97, 326-329.

Van de Wiel, B. J. H., R. J. Ronda, A. F. Moene, H. A. R. De Bruin, and A. A. M. Holtslag, 2002a: Intermittent turbulence and oscillations in the stable boundary layer. Part I: A bulk model. J. Atmos. Sci., 59, 942-958.

- A. F. Moene, R. J. Ronda, H. A. R. De Bruin, and A. A. M. Holtslag, 2002b: Intermittent turbulence and oscillations in the stable boundary layer. Part II: A system dynamics approach. J. Atmos. Sci., 59, 2567-2581.

O. K. Hartogensis, H. A. R. De Bruin, and A. A. M. Holtslag, 2003: Intermittent turbulence and oscillations in the stable boundary layer. Part III: A classification for observations during CASES-99. J. Atmos. Sci., 60, 25092522 .

,- , G. J. Steeneveld, O. K. Hartogensis, and A. A. M. Holtslag, 2007: Predicting the collapse of turbulence in stably stratified boundary layers. Flow Turbul. Combust., 79, 251274.

- — - and H. J. J. Jonker, 2012: The cessation of continuous turbulence as precursor of the very stable nocturnal boundary layer. J. Atmos. Sci., 69, 3097-3115.

Van Ulden, A. P., and J. Wieringa, 1996: Atmospheric boundary layer research at Cabauw. Bound.-Layer Meteor., 78, 39-69.

Walters, J. T., R. T. McNider, X. Shi, W. B. Norris, and J. R. Christy, 2007: Positive surface temperature feedback in the stable nocturnal boundary layer. Geophys. Res. Lett., 34, L12709, doi:10.1029/2007GL029505.

Wieringa, J., 1989: Shapes of annual frequency distributions of wind speed observed on high meteorological masts. Bound.Layer Meteor., 47, 85-110.

Wyngaard, J. C., 2010: Turbulence in the Atmosphere. Cambridge University Press, 393 pp.

Zhou, B., and F. K. Chow, 2011: Large-eddy simulation of the stable boundary layer with explicit filtering and reconstruction turbulence modeling. J. Atmos. Sci., 68, 2142-2155.

Zilitinkevich, S. S., T. Elperin, N. Kleeorin, I. Rogachevskii, I. Esau, T. Mauritsen, and M. W. Miles, 2008: Turbulent energetics in stably stratified geophysical flows: Strong and weak mixing regimes. Quart. J. Roy. Meteor. Soc., 134, 793-799. 